\title{
Factors associated with body image among patients with an implanted left ventricular assist device
}

Semyon Melnikov, Miri Abuhazira, Dimitry Golobov, Victoria Yaari, Tiny Jaarsma and Tuvia Ben Gal

The self-archived postprint version of this journal article is available at Linköping University Institutional Repository (DiVA):

http://urn.kb.se/resolve?urn=urn:nbn:se:liu:diva-172434

N.B.: When citing this work, cite the original publication.

Melnikov, S., Abuhazira, M., Golobov, D., Yaari, V., Jaarsma, T., Ben Gal, T., (2020), Factors associated with body image among patients with an implanted left ventricular assist device, Heart \& Lung, 49(6), 803-807. https://doi.org/10.1016/j.hrtlng.2020.08.022

Original publication available at:

https://doi.org/10.1016/j.hrtlng.2020.08.022

Copyright: Elsevier

http://www.elsevier.com/

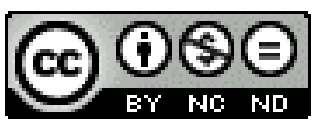




\section{Highlights}

- Among LVAD patients, body image is associated with sexual functioning, LVAD confidence, and well-being.

- Sexual functioning and LVAD confidence predicted LVAD patients' body image. 
Factors associated with body image among patients with an implanted Left Ventricular Assist Device

\begin{abstract}
Background

Among Left Ventricular Assist Device (LVAD) implanted patients, body image modifications might occur.

\section{Objectives}

To explore the associations between sexual functioning, confidence in LVAD technology, personal wellbeing, and body image.

\title{
Methods
}

The cross-sectional study included 30 conveniently recruited LVAD implanted patients who completed Body Image Scale, sexual functioning, confidence in LVAD technology, and Personal Wellbeing Index questionnaires. The associations between the research variables were examined with multiple regression analyses.

\section{Results}

The mean age of the participants was $63(\mathrm{SD}=10), 90 \%$ were men. The mean scores were: sexual functioning - $2.43(\mathrm{SD}=1.20$, range 1-5); confidence in LVAD technology - 2.63 $(\mathrm{SD}=1.04$, range 1-5); Well-Being Index - $6.61(\mathrm{SD}=1.53$, range $0-10)$; and body image 14.5 $(\mathrm{SD}=4.17$, range 5-20). Sexual functioning $(\mathrm{p}<.01)$ and LVAD technology confidence $(\mathrm{p}=.04)$ significantly predicted body image.

\section{Conclusions}

Healthcare professionals should be aware of challenges regarding body image faced by LVAD implanted patients and to address related factors. 
Key words: body image, sexual functioning, confidence in LVAD technology Introduction

Heart failure (HF) is a global public health problem that affects more than 26 million people worldwide. ${ }^{1}$ Heart transplantation (HTx) has been the treatment of choice for endstage HF patients but is extremely limited by the low availability of suitable heart donors. ${ }^{2}$ Implantable Left Ventricular Assist Devices (LVADs) have been used for decades in very advanced HF or cardiogenic shock primarily as a bridge to heart transplantation (BTT). Improvements in LVAD technology and in the survival of LVAD supported patients led to more extensive use of LVADs also among patients with relative contraindications to HTx as a bridge-to-decision (BTD) option or as a permanent treatment: destination therapy (DT). ${ }^{3}$ LVADs have been demonstrated to prolong HF patients' survival and to improve their quality of life. ${ }^{4}$

LVAD implantation has been demonstrated to have a considerable effect on patients' body image. Patients feel it hard to accept the device being an extension of their body and spoke about their bodies changing during the time they had been supported with the LVAD. ${ }^{5}$ Following implantation, patients experienced their body as different, were disgusted by their own body, felt ashamed by their changed appearance, and felt that their body had been invaded. ${ }^{6}$ On discharge from the hospital, along with the positive outcomes of the LVAD support, patients reported feelings of awe and dismay when looking at themselves in the mirror. ${ }^{7}$ Almost half (47\%) the patients felt that the LVAD negatively affected their selfimage, which more than half thought was important for sexual satisfaction. ${ }^{8}$

LVAD implantation can significantly affect patients' sexual function. In a study of 301 LVAD implanted patients (24\% women), more than half the participants reported a decline in sexual desire, arousal, and orgasm post-LVAD implantation. ${ }^{8}$ In a study that compared sexual function among HTx and LVAD implanted patients, pleasure and 
satisfaction with sex were significantly lower among LVAD implanted patients. The specific problems in sexual function included erectile failure and vaginal dryness, difficulties with the device, external batteries, or cable, and problems reaching orgasm. ${ }^{9}$ Following LVAD implantation, a remarkable decrease in the degree of satisfaction and in sexual activity frequency were related to avoiding a partner's disappointment, as well as fear of sudden cardiac arrest and of LVAD failure during sexual activity. ${ }^{10}$

Fear of LVAD failure, trust and confidence in the LVAD were among the subthemes identified in interviews with LVAD implanted patients. ${ }^{5}$ Distrust in LVAD technology was among the common reasons for LVAD implantation decline. ${ }^{11}$ Decliners referred to the LVAD as "experimental," and "a scientific project" owing to their lack of familiarity or comfort with LVAD technology. ${ }^{11}$ Among young adults living with mechanical circulatory support, feeling trust and confidence in the ventricular assist device and seeing it as a friend were among the main themes identified. ${ }^{6}$

A systematic review by Adams et al. (2015) reported on LVAD ability to improve patients' quality of life. ${ }^{12}$ Among LVAD supported patients with totally implantable or extracorporeal LVAD, quality of life scores were highest among the totally implantable group. ${ }^{13,14}$ At the same time, in a study on 61 LVAD implanted patients participating in cardiac rehabilitation, $30 \%(\mathrm{n}=18)$ demonstrated lower quality of life scores that were associated with long-term mortality. ${ }^{15}$

The associations between sexual functioning, confidence in LVAD technology, personal wellbeing, and body image are not yet fully understood. The purpose of the current study was to examine the relationships between sexual functioning, confidence in LVAD technology, and personal wellbeing with body image among LVAD implanted patients.

\section{Materials and Methods}

\section{Design}


Cross-sectional survey. The ethical committee of the medical center at which the study was conducted approved the study.

\section{Setting}

The participants were recruited from a LVAD implanted cohort at a Cardiothoracic Surgery Department in a tertiary medical center in central Israel. Recruitment took place between October 2017 and February 2018.

\section{Population}

The convenience sample consisted of 34 potential eligible participants who were approached by two research nurses routinely caring LVAD supported patients. The nurses invited patients to participate in a study aimed at exploring the experience of patients with an implanted LVAD. All the participants were Hebrew speaking, aged 18 or older, with no evidence of cognitive impairment, and able to sign the informed consent form. Participants were assured that if they refused to take part in the study, their care and their relationship with the clinicians would not be affected in any way, and that all information would remain confidential. Two potential participants were not qualified since they were not able to read Hebrew. Those who signed the informed consent form received the questionnaire to fill out. Filling out the questionnaire took about 20 minutes. For unclear reasons, two patients declined to fill out the questionnaire. A final sample size consisted of 30 patients (response rate $88.2 \%$ ) (Table 1$)$.

\section{Measures}

A self-report questionnaire consisted five sections: (i) Body Image Scale (BIS), (ii) Sexual functioning, (iii) Confidence in LVAD technology, (iv) Personal Wellbeing Index, and (v) sociodemographic and clinical characteristics.

(i) The participants' perceived body image was examined by the Body Image Scale (BIS) with an adequate internal consistency. ${ }^{16}$ The BIS consisted of five questions measuring the 
perception and satisfaction with his or her body following the implantation of LVAD. Patients ranked items on a scale ranging from 1 (No, not at all) to 4 (Yes, extremely), resulting in a total score with a minimum of 5 and a maximum of 20 (reverse scored). Examples of the questions are: "Do you feel less attractive as a result of your operation?" and “Do you feel less feminine/masculine as a result of your operation?" Higher BIS scores represented a more positive perception of body image. In the present study, the internal reliability was 0.79 .

(ii) Sexual functioning was assessed on a 5-point scale asking participants: "Overall, how would you rate your ability to function sexually during the last four weeks?"17 The response choices included: 1 (very poor), 2 (poor), 3 (fair), 4 (good), and 5 (very good), with higher scores representing higher sexual functioning. Sexual dysfunction has been defined as a score of 1 or 2, while appropriate sexual functioning has been defined as a score of 3, 4, or 5 . (iii) "Confidence in LVAD technology" was measured by a scale designed by the current authors based on literature review. The scale contained 10 items. Participants responded on a scale ranging from 1 (strongly disagree) to 5 (strongly agree) (reverse scored). The higher the mean score the higher the confidence in LVAD technology. Examples of the items are: "I'm worried about the battery shutting down", "In case of a severe blow the device may stop working," "I'm afraid the device's lines will detach from my body." Cronbach's alpha for the scale was .89. In order to empirically test the factor structure of the questionnaire, exploratory factor analysis (EFA) was performed. The data were shown to be appropriate for EFA $(\mathrm{KMO}=.696$, Bartlett's Test of Sphericity $=P<.001)$. Principal Component Analysis with promax rotation indicated two factors with an eigenvalue greater than 1 (5.16 and 1.42). These two factors explained $65.81 \%$ of the variance. Based on the rotated component matrix, the items had significant factor loading, ranging from .535 to .884 . In the two-factor structure Factor 1, labeled "Fears of technical malfunctions in the device", included item 2 (In case of 
a severe blow the device may stop working), item 3 (The device may stop working while replacing the battery), item 4 (I'm afraid that if a device will malfunction they won't help me), and item 8 (I'm afraid the device's lines will detach from my body). Factor 2, labeled "Adjustments in everyday life" included item 1 ("I'm worried about the battery shutting down"), item 5 ("Because of the fear of device failure, I prefer to stay at home"), item 6 ("In crowded places the device may be pulled off'), item 7 (There is a high risk of contamination of the lines entering my body"), item 9 (Because of the fear of wetting the device, I rarely shower), and item 10 (I'm afraid a thief will tear my bag with the device when I am outside). Cronbach's alpha for Factor 1 ("Fears of technical malfunctions in the device") was 0.89, for Factor 2 ("Adjustments in everyday life") 0.79, and for the whole scale 0.89.

(iv) Subjective wellbeing was measured by the Personal Wellbeing Index (PWI). ${ }^{18}$ The scale askes how satisfied people are with seven life domains. The domains include standard of living, personal health, achievement in life, personal safety, personal relationships, community connectedness, and future security. An 11-point, end-defined Likert scale was used $(0=$ extremely dissatisfied to $10=$ extremely satisfied $)$. The mean of the seven domains forms the PWI score, with a higher score indicating higher personal wellbeing. In the present study, internal reliability was $\alpha=.89$.

(v) Sociodemographic and clinical characteristics included participants' age, gender, marital status, time since device implantation, heart failure etiology, LVAD indication, complications after LVAD implantation, and medications.

\section{Statistical analysis}

Mean and standard deviation, median, and interquartile range were used to describe the sample and the variables included in the analysis. To analyze the relationships between continuous variables (sexual functioning, confidence in LVAD technology, Well-Being Index, and body image), Pearson correlations were used. T-test analysis was used to compare 
means between groups. Multivariate linear regression was used to explore the variance in body image explained by independent variables (sexual functioning, confidence in LVAD technology, and Well-Being Index) (Fig. 1). Preliminary analyses were conducted to ensure no violation of the assumptions of normality, linearity, multicollinearity, autocorrelation, homoscedasticity, and the absence of influential values.

IBM SPSS Statistics version 25 (IBM Corp., Armonk, New York) was used to analyze the data.

\section{Results}

\section{Descriptive data and differences among groups}

The mean age of the participants was $63(\mathrm{SD}=10)$, ranging from 39 to 80 , and $90 \%$ $(n=27)$ of the participants were men (Table 1). The mean time from LVAD implantation was $29(\mathrm{SD}=20)$ months. Ischemic cardiomyopathy was the etiology of heart failure among 93\% $(n=28)$ of the participants, while among $7 \%(n=2)$ the etiology was non-ischemic cardiomyopathy. Indications for LVAD implantation were bridge to transplantation, $77 \%$ $(n=23)$, destination therapy, $20 \%(n=6)$, and bridge to recovery, $3 \%(n=1)$. The mean scores of the main research variables were as follows: sexual functioning - $2.43(\mathrm{SD}=1.20$, range 15); confidence in LVAD technology - 2.63 ( $\mathrm{SD}=1.04$, range 1-5); Well-Being Index - 6.61 $(\mathrm{SD}=1.53$, range $0-10)$; and body image $-14.5(\mathrm{SD}=4.17$, range 5-20). No significant age differences were found between respondents with appropriate sexual functioning $[\mathrm{M}=60.7$ $(\mathrm{SD}=10.5)]$ and those with sexual dysfunction $[\mathrm{M}=64.8,(\mathrm{SD}=9.8)],(\mathrm{t}=1.0, \mathrm{p}=.31)$, respectively. Participants who had a partner reported higher levels of personal wellbeing $(\mathrm{M}=7.36[\mathrm{SD}=.60]$ vs. $\mathrm{M}=6.42[\mathrm{SD}=1.64])$, than participants without a partner $(\mathrm{t}=2.26$, $p=.03$ ). No difference in sexual functioning was found between participants with and without a partner.

\section{Relationships among variables}


There was a statistically significant positive correlation between sexual functioning, LVAD technology confidence, subjective wellbeing and perception of body image $(\mathrm{r}=.59$, $\mathrm{p}<.01 ; \mathrm{r}=.40, \mathrm{p}<.05 ; \mathrm{r}=.37, \mathrm{p}<.05$, respectively) (Table 2). In other words, participants with higher sexual functioning, higher confidence in LVAD technology, or a higher score on the Well-Being Index reported a better perception of their body image. Moreover, a negative correlation was found between body image scores and the difference in BMI before LVAD implantation and in the last six months $(\mathrm{r}=-.38, \mathrm{p}=.04)$. In other words, a higher increase in BMI was associated with a lower body image.

\section{Regression analysis}

To measure the unique predictive contribution of selected variables to the perceived body image of LVAD supported patients, a multiple linear regression analysis was performed. All the research variables were included as independent variables and perceived body image as the dependent variable. This analysis demonstrated that sexual functioning $(t=3.35, \beta=.49, b=1.75, p<.01)$ and confidence in LVAD technology $(t=2.15, \beta=.31, b=1.29$, $p=.04)$ significantly predicted body image (Table 3 ). The main research variables together explained $52 \%$ of the variance in the Body Image variable among patients implanted with an LVAD

\section{Discussion}

The aim of the research was to examine factors related to the perception of body image among patients with an implanted LVAD. The results of the current pilot work show that sexual functioning, confidence in LVAD technology, and personal well-being were positively associated with perceived body image among LVAD implanted patients, with sexual functioning and confidence in LVAD technology significantly predicting body image. Future studies with a sufficiently large sample will be able to examine the effects of all the independent variables and possible confounders in the hierarchical regression analysis. 
In the current study, participants who had higher sexual functioning reported higher body image scores. Problems with self-image have previously been reported as contributing to problems in sexual function and performance among LVAD implanted patients. ${ }^{9}$ LVAD implantation can negatively affect patients' body image, having an effect on sexual satisfaction. ${ }^{8}$ In the current study, these relationships were explored from another perspective, namely, that body image is explained by sexual function. The authors contend that the relationship between sexual functioning and body image is more complex than simply a onedirection relationship, similar to the relationship between depression and sexual functioning. Depression might lead to lower sexual function on one hand, and on the other, sexual dysfunction might lead to depression. Depression is characterized by a constellation of symptoms such as reduced energy, loss of interest, decreased self-esteem, producing differences in sexual relationships. ${ }^{19}$ Conversely, a decrease in sexual desire may precede the onset of depression. ${ }^{20}$ Similarly, a decrease in sexual functioning among LVAD implanted patients may lead to lower body image and, conversely, lower body image may lead to a decrease in sexual functioning. The current results demonstrated a negative association between BMI and body image, with a higher increase in BMI associated with lower body image. A previous study demonstrated that body image satisfaction mediated the relationships between degree of overweight and depression/self-esteem. ${ }^{21}$ Similarly, in the current study, lower Body Image scores may be related to weight gain following LVAD implantation.

LVAD implantation itself may also have an independent impact on both body image and sexual functioning. LVAD implantation has been found to negatively affect patients' body image perception. ${ }^{5,8}$ Patients reportedly feel feelings of awe and dismay when looking at themselves in the mirror ${ }^{7}$ and try to improve their own self-image by accommodating the LVAD. ${ }^{22}$ On the other hand, following LVAD implantation patients reported improvement in 
sexual activities ${ }^{23,24}$ or prominent sexual dysfunction ${ }^{9}$ Among the problems in sexual function reported by LVAD implanted patients were: erectile failure and vaginal dryness, difficulties with the device, external batteries, or cable, problems reaching orgasm, fear of harming themselves, depression, pain, issues with self-image, and others. ${ }^{9}$

In the current study, positive relationships were found between body image and confidence in LVAD technology. As previously reported, among LVAD implanted patients body image concerns were associated with device related distress. ${ }^{25}$ The current results are also similar to those reported by Bruce et al. (2015) who showed that among the reasons that eligible candidates declined LVAD implantation was importance of bodily integrity. ${ }^{11}$ Patients expressed disdain for being "opened up", appealing to a desire to preserve their body integrity. ${ }^{11}$ Among individuals surviving sudden cardiac arrest and living with an implantable cardioverter defibrillator (ICD), ICD implantation affected patients' body image, changing their sense of self so that the ICD becomes a part of their body. Patients reported personalization of the device, expressed by naming it "sparky", "zapper", "old boy", or "my own paramedic. ${ }^{26}$ In the current study, higher LVAD confidence levels correlating with better body image scores may mean better acceptance of the device and lower device related distress. In the current authors' opinion, the findings regarding a positive association between confidence in the LVAD and body image are novel.

In addition, an association was found between body image and personal wellbeing. This corroborates findings by Tosto et al. (2019), who reported that lower scores of body image were associated with poorer quality of life and lower levels of device acceptance among LVAD implanted patients. While not examined in the current study, poorer body image may affect patient acceptance of the LVAD. The current results highlight the importance of the body image variable as affecting the personal wellbeing of LVAD 
implanted patients. Future studies should address the optimal content and timing of counseling for patients and caregivers to improve their outcomes. ${ }^{14}$

\section{Limitations}

The present study has several limitations. The major limitation of the current study is its extremely small sample size, determined by the inclusion of patients with an implanted LVAD but only from a single medical center. Second, it was a cross-sectional design study, with no cause and effect relationship established. Third, the vast majority of participants in the current sample were men. Among women, the relationships between the variables examined may be different. Additional limitations of the current study are related to the instruments. Among the limitations of the sexual functioning instrument were that it had a single item and its original use was in a male-only sample. Among the limitations of the "Confidence in LVAD technology" scale were that it was developed by the investigators and has had no formal evaluation of validity. Moreover, the association between body image and other main variables should be treated with caution, given the small sample size and therefore the inability to address the potential confounders.

\section{Conclusions}

This study shows that body image is associated with sexual functioning, confidence in LVAD technology, and personal well-being of patients with an implanted LVAD. Sexual functioning and LVAD technology confidence predicted body image. The current results highlight the importance of raising by the healthcare staff the issue of LVAD implantation's effect on body image, along with examining variables such as sexual function, confidence in the LVAD technology, and personal wellbeing. 


\section{Bibliography}

1. Bloom MW, Greenberg B, Jaarsma T, et al. Heart failure with reduced ejection fraction. Nat Rev Dis Primers. 2017;3:17058. doi:10.1038/nrdp.2017.58

2. Cai AW, Islam S, Hankins SR, Fischer W, Eisen HJ. Mechanical circulatory support in the treatment of advanced heart failure. Am J Transplant. 2017;17(12):3020-3032. doi:10.1111/ajt.14403

3. Gustafsson F, Rogers JG. Left ventricular assist device therapy in advanced heart failure: patient selection and outcomes. Eur J Heart Fail. 2017;19(5):595-602. doi:10.1002/ejhf.779

4. Maciver J, Ross HJ. Quality of life and left ventricular assist device support. Circulation. 2012;126(7):866-874. doi:10.1161/CIRCULATIONAHA.111.040279

5. Chapman E, Parameshwar J, Jenkins D, Large S, Tsui S. Psychosocial issues for patients with ventricular assist devices: a qualitative pilot study. Am J Crit Care. 2007;16(1):72-81.

6. Lachonius M, Hederstedt K, Axelsson ÅB. Young adult patients' experience of living with mechanical circulatory support: A phenomenological hermeneutical study. Nurs Open. 2019;6(2):651-658. doi:10.1002/nop2.247

7. Casida JM, Marcuccilli L, Peters RM, Wright S. Lifestyle adjustments of adults with long-term implantable left ventricular assist devices: a phenomenologic inquiry. Heart Lung. 2011;40(6):511-520. doi:10.1016/j.hrtlng.2011.05.002

8. Eckman PM, Dhungel V, Mandras S, et al. Sexual function after left ventricular assist device. J Am Coll Cardiol. 2013;61(19):2021-2022. doi:10.1016/j.jacc.2013.02.022

9. Hasin T, Jaarsma T, Murninkas D, et al. Sexual function in patients supported with left ventricular assist device and with heart transplant. ESC Heart Fail. 2014;1(2):103-109. doi:10.1002/ehf2.12014

10. Merle P, Maxhera B, Albert A, et al. Sexual concerns of patients with implantable left ventricular assist devices. Artif Organs. 2015;39(8):664-669. doi:10.1111/aor.12535

11. Bruce CR, Kostick KM, Delgado ED, et al. Reasons why eligible candidates decline left ventricular assist device placement. J Card Fail. 2015;21(10):835-839. doi:10.1016/j.cardfail.2015.06.008

12. Adams EE, Wrightson ML. Quality of life with an LVAD: A misunderstood concept. Heart Lung. 2018;47(3):177-183. doi:10.1016/j.hrtlng.2018.02.003

13. Kato N, Jaarsma T, Ben Gal T. Learning self-care after left ventricular assist device implantation. Curr Heart Fail Rep. 2014;11(3):290-298. doi:10.1007/s11897-0140201-0

14. Kato NP, Okada I, Imamura T, et al. Quality of life and influential factors in patients implanted with a left ventricular assist device. Circ J. 2015;79(10):2186-2192. doi:10.1253/circj.CJ-15-0502 
15. Modica M, Minotti A, De Maria R, et al. Coping, mood, quality of life, and outcomes in recipients of left ventricular assist devices: A cluster analysis. Psychosom Med. 2019;81(2):192-199. doi:10.1097/PSY.0000000000000658

16. Dunker MS, Stiggelbout AM, van Hogezand RA, Ringers J, Griffioen G, Bemelman WA. Cosmesis and body image after laparoscopic-assisted and open ileocolic resection for Crohn's disease. Surg Endosc. 1998;12(11):1334-1340.

17. Dahn JR, Penedo FJ, Gonzalez JS, et al. Sexual functioning and quality of life after prostate cancer treatment: considering sexual desire. Urology. 2004;63(2):273-277. doi:10.1016/j.urology.2003.09.048

18. Cummins R, Eckersley R, Pallant J, van Vugt J, Misajon R. Developing a National Index of Subjective Wellbeing: The Australian Unity Wellbeing Index. Social Indicators Research. 2003;64(2):159-190.

19. Baldwin DS. Depression and sexual dysfunction. Br Med Bull. 2001;57:81-99. doi:10.1093/bmb/57.1.81

20. Schreiner-Engel P, Schiavi RC. Lifetime psychopathology in individuals with low sexual desire. J Nerv Ment Dis. 1986;174(11):646-651. doi:10.1097/00005053198611000-00002

21. Friedman KE, Reichmann SK, Costanzo PR, Musante GJ. Body image partially mediates the relationship between obesity and psychological distress. Obes Res. 2002;10(1):33-41. doi:10.1038/oby.2002.5

22. Sandau KE, Hoglund BA, Weaver CE, Boisjolie C, Feldman D. A conceptual definition of quality of life with a left ventricular assist device: results from a qualitative study. Heart Lung. 2014;43(1):32-40. doi:10.1016/j.hrtlng.2013.09.004

23. Samuels LE, Holmes EC, Petrucci R. Psychosocial and sexual concerns of patients with implantable left ventricular assist devices: a pilot study. $J$ Thorac Cardiovasc Surg. 2004;127(5):1432-1435. doi:10.1016/j.jtcvs.2003.12.009

24. Marcuccilli L, Casida JJ, Peters RM, Wright S. Sex and intimacy among patients with implantable left-ventricular assist devices. J Cardiovasc Nurs. 2011;26(6):504-511. doi:10.1097/JCN.0b013e31820e2fae

25. Tosto C, Adamo L, Craddock H, et al. Relationship between device acceptance and patient-reported outcomes in Left Ventricular Assist Device (LVAD) recipients. Sci Rep. 2019;9(1):10778. doi:10.1038/s41598-019-47324-z

26. Dickerson SS. Redefining life while forestalling death: living with an implantable cardioverter defibrillator after a sudden cardiac death experience. Qual Health Res. 2002;12(3):360-372. doi:10.1177/104973202129119946 
Table 1: Sample characteristics, $(\mathrm{N}=30)$

\begin{tabular}{|c|c|}
\hline Variable & \\
\hline $\begin{array}{l}\text { Age (years), mean (SD), } \\
\text { min-max }\end{array}$ & $63.1(10.1), 39-80$ \\
\hline Male gender, $\mathrm{n}(\%)$ & $27(90)$ \\
\hline Marital status & \\
\hline Married/living with partner $\mathrm{n}(\%)$ & $24(80)$ \\
\hline $\begin{array}{l}\text { Time since device implant (month), } \\
\text { mean (SD), median, IQR, min-max }\end{array}$ & $28.7(20.2), 23.5,29.8,2-72$ \\
\hline Heart failure etiology, n (\%) & \\
\hline Ischemic cardiomyopathy & $28(93.3)$ \\
\hline Non-ischemic cardiomyopathy & $2(6.7)$ \\
\hline LVAD indication, $\mathrm{n}(\%)$ & \\
\hline BTT & $23(76.7)$ \\
\hline DT & $6(20)$ \\
\hline BTR & $1(3.3)$ \\
\hline Complications after LVAD* & \\
\hline Stroke & $3(10)$ \\
\hline ESRD & $2(6.7)$ \\
\hline Infection & $6(20)$ \\
\hline GIB & $4(13.3)$ \\
\hline Medications & \\
\hline Antiplatelets & $28(93.3)$ \\
\hline Anticoagulants & $30(100)$ \\
\hline
\end{tabular}




\begin{tabular}{|l|l|}
\hline Beta Blockers & $25(83.3)$ \\
Diuretics & $6(20)$ \\
ACE Inhibitors & $10(33.3)$ \\
Antidepressants (SSRIs) & $8(26.7)$ \\
\hline $\begin{array}{l}\text { BMI, mean (SD), min-max, range } \\
\text { Before the implantation }\end{array}$ & $26.0(5.0), 18.6-39.9,21.3$ \\
During the last six months & $27.8(4.9), 20.5-41.1,20.6$ \\
Difference in BMI before & \\
implantation and in the last six & \\
months, average (SD), min-max, & \\
range
\end{tabular}

There may be more than one complication in one patient

BTT - Bridge to transplantation, DT - Destination therapy, BTR - Bridge to recovery, NYHA -

New York Heart Association, ESRD - End stage renal disease, GIB - Gastrointestinal bleeding, ACE -Angiotensin converting enzyme, SSRIs - Selective serotonin reuptake inhibitors

Table 2: Correlation analysis of research variables (Pearson's correlation)

\begin{tabular}{|l|l|l|l|l|l|}
\hline & Variable & $\mathbf{1}$ & $\mathbf{2}$ & $\mathbf{3}$ & $\mathbf{4}$ \\
\hline 1. & Body image $(\mathrm{n}=30)$ & - & & & \\
\hline 2. & Sexual functioning $(\mathrm{n}=28)$ & $.59^{* *}$ & - & & \\
\hline 3. & LVAD technology confidence $(\mathrm{n}=30)$ & $.40^{*}$ & -.18 & - & \\
\hline 4. & Well-Being Index $(\mathrm{n}=30)$ & $.37^{*}$ & .17 & -.17 & - \\
\hline
\end{tabular}

$* \mathrm{p}<.05, * * \mathrm{p}<.01$ 
Table 3. Main effects on body image.

\begin{tabular}{|l|l|l|l|l|l|l|}
\hline Characteristic & $\mathbf{b}$ & SE & $\boldsymbol{\beta}$ & $\mathbf{t}$ & $\mathbf{p}$ & $\mathbf{9 5 \%}$ CI* \\
\hline Sexual functioning & 1.75 & .52 & .49 & 3.35 & $<.01$ & $.67-2.82$ \\
\hline Confidence in LVAD & 1.29 & .60 & .31 & 2.15 & .04 & $.05-2.54$ \\
technology & & & & & & \\
\hline Well-Being Index & .77 & .40 & .28 & 1.91 & .07 & $-.06-1.6$ \\
\hline
\end{tabular}

$\mathrm{R}^{2}=.52, \mathrm{~F}=8.79, \mathrm{p}<.001$

${ }^{*} \mathrm{CI}-$ confidence interval

Figure 1. Research model

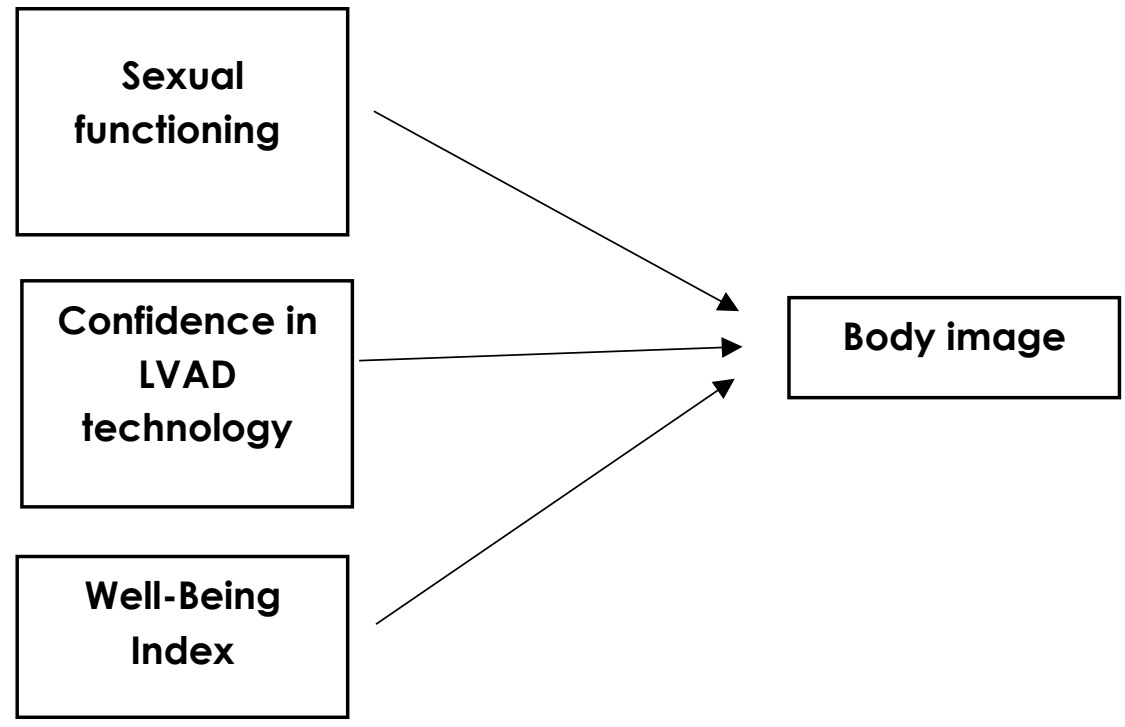


